\title{
High-Risk Behaviors During Incarceration in African-American Men Treated for HIV at Three Los Angeles Public Medical Centers
}

\author{
*Amy Rock Wohl, *Denise Johnson, †Wilbert Jordan, *Sharon Lu, ‡Gildon Beall, \\ $\S$ Judith Currier, and *Peter R. Kerndt
}

*HIV Epidemiology Program, Los Angeles County Department of Health Services; †Martin Luther King-Drew Medical Center; $\$$ Harbor-UCLA Medical Center; and §Los Angeles County-University of Southern California Medical Center and University of California Los Angeles CARE Center, Los Angeles, California, U.S.A.

\begin{abstract}
Objectives: This paper describes research that examined the association between high-risk sexual and drug-using behaviors during incarceration and HIV infection for African-American men receiving HIV care at three public medical centers in Los Angeles County (LAC), California.

Methods: A case-control study was conducted in which $305 \mathrm{HIV}$ - infected AfricanAmerican men and 305 neighborhood controls, ages 20 to 49, were frequency-matched by age.

Results: After controlling for anal sex while not incarcerated, we found no association between anal sex during incarceration and HIV (odds ratio [OR], 1.1; 95\% confidence interval $[\mathrm{CI}], 0.6-2.2)$. Among men with a history of incarceration $(n=$ $332)$, the percentage reporting anal sex with men outside of incarceration (45\%) was greater than those reporting anal sex while incarcerated (16\%). Injection drug use (IDU) during incarceration was also not associated with HIV when controlling for IDU outside of incarceration (OR, 1.6; 95\% CI, 0.5-4.9). Increased time in jail or prison was associated with less HIV infection $(p=.001)$.

Conclusions: Although high-risk behaviors are more common in the community than in the incarcerated setting for this study group, incarcerated populations represent a high-risk group for whom access to prevention messages is limited. Periods of incarceration represent a unique opportunity to convey prevention messages that focus on high-risk behaviors outside the incarcerated setting.

Key Words: Prisons-Sexual behaviors—Drug users—Risk factors-Heterosexual men-Homosexual men-Epidemiology
\end{abstract}

African-American men have had the highest AIDS rate of any racial/gender subgroup in Los Angeles County (LAC) since 1988 (1). Most (67\%) HIV infections among African-American men with AIDS reported through 1998 in LAC have been acquired through maleto-male sex (MSM); $12 \%$ is associated with injection drug use (IDU); and 9\% have been among men who

Address correspondence and reprint requests to Amy Rock Wohl, HIV Epidemiology Program, 600 South Commonwealth Avenue, Suite 805, Los Angeles, CA 90005, U.S.A.; email: awohl@dhs.co.la.ca.us.

Manuscript received October 4, 1999; accepted March 23, 2000. practice both MSM and IDU (1). African-American men comprised $31 \%$ of California's prison population in 1998 (2) and $8 \%$ of the state's male population in the same year (3). It has been theorized that the elevated AIDS rate in African-American men is due in part to high rates of incarceration and, therefore, greater exposure to unprotected sex with men with high HIV seroprevalence (4-6). HIV seroprevalence was $2.7 \%$ among new entrants into the LAC jail system in 1995 (7) and 2.4\% among a sample of men entering the California prison system in 1994 (8). 
Research on incarcerated persons in other locations suggests that IDU during incarceration is fairly common $(9,10)$ and is associated with increased HIV risk $(11,12)$. Sexual behaviors during incarceration, however, have not been widely documented (10-13) and have not been linked to increased HIV risk (11-13). There have been limitations, however, to previous research that have included the collection of risk behavior data in jail or prison settings (10-13) where a person may be unlikely to admit to illegal drug or sexual behaviors $(5,11,14)$ and small numbers of persons reporting sex while incarcerated (10-13). In addition, none of the earlier studies focused exclusively on African-American men who are overrepresented among the incarcerated (2) and among persons with AIDS (1). Finally, there are very limited data on high-risk sexual and drug-using behaviors in the California prison and Los Angeles County jail systems.

To describe high-risk behaviors in the incarcerated setting and examine the contribution of these behaviors to HIV risk for African-American men, HIV-infected men were recruited from county health facilities where data on risk behaviors during incarceration were collected postincarceration to minimize underreporting. Data on high-risk behaviors among incarcerated populations are needed to further develop effective HIV prevention programs within correctional systems.

\section{METHODS}

We conducted a case-control study in which 305 HIV-infected African-American men, ages 20 to 49 , were identified at three large county medical facilities that contributed $38 \%$ of all AIDS cases diagnosed among African-American men in LAC through September 1996. Eligible cases included any 20- to 49-year-old African-American man infected with HIV who was an active patient at one of these three medical facilities during the recruitment period, January, 1997 through October, 1998. Eligible cases were approached for recruitment into the study by letter, telephone, and in person at the time of a clinic appointment. Each case was matched to a control by age (frequency matching) and neighborhood of residence within 10 blocks. All potential controls were invited to participate and were recruited from public areas that included parks, coffee shops, and street corners. Controls were also recruited through hand-out information sheets (flyers) that were posted in the neighborhoods of the study participants. All controls were administered an oral test for the detection of HIV antibodies. Sixteen persons who tested positive for HIV were not eligible as controls and were thereby referred to medical and social support services. The response rate was $47 \%$ for the cases and $82 \%$ for the controls.

All participants were administered a detailed questionnaire on demographics, incarceration histories, and high-risk sexual and drugusing behaviors occurring inside and outside of jail or prison, sexually transmitted disease (STD) history, health care use, and social support. Participants were asked about high-risk sexual and drug-using behaviors occurring between 1978, the approximate time that HIV became prevalent in the United States, and the person's first positive HIV test result. Controls were asked about the time period corresponding to
1978 and the first positive HIV test result of his matched case. Study interviewers reflected the race and gender of study participants, a strategy supported in the literature (15) and recommended by local focus group participants. The study interviewers were two African-American men with extensive experience interviewing men of color regarding sexual and drug-use behaviors.

Institutional review board approval was obtained from all three study recruitment sites before collection of data and written informed consent was completed by each participant before administration of the study questionnaire. All participants received a $\$ 30$ U.S. incentive fee following completion of the questionnaire.

To assess the generalizability of the study group to HIV-infected African-American men seen at each of three clinics, demographic characteristics of the study group were compared with those of a population-based sample of African-American male participants in the U.S. Centers for Disease Control and Prevention (CDC)'s Supplement to HIV/AIDS Surveillance Project (SHAS) who were diagnosed with AIDS at each of the three sites from 1990 to 1999 (16).

Univariate analyses are presented and odds ratios (ORs) and 95\% confidence intervals (95\% CIs) were calculated and adjusted for confounding, as needed. A $\chi^{2}$ test for trend was used to examine for dose-response trends in the data. All analyses were conducted using SAS software (17).

\section{RESULTS}

Demographic data on the cases and controls are presented in Table 1. Cases were more likely to be single, unemployed, and on public assistance. Most cases and controls had at least a high-school education, and $69 \%$ of the cases self-identified as gay or bisexual compared with $11 \%$ of the controls. The mean age of the cases and controls was 38. Of 280 (92\%) HIV-infected persons with available medical records, $42 \%$ had progressed to AIDS. Among HIV-infected persons, $66 \%$ had their HIV infection diagnosed between 1991 and 1998.

Data on incarceration histories are presented in Table 2. More controls (59\%) than cases (50\%) reported a history of incarceration; however, this difference was not statistically significant. Of those with a history of any previous incarceration, most cases $(56 \%)$ and controls $(54 \%)$ had been incarcerated once or twice. A large proportion of cases (51\%) and controls (39\%) who had been incarcerated were incarcerated a total of 6 months or less. There was a decreasing risk for HIV associated with an increase in the number of months incarcerated in jail or prison $(p=.001)$. Those with prison time in addition to jail time were at no greater risk for HIV than persons with only a history of incarceration in a jail (OR, 0.8; 95\% CI, 0.5-1.2).

Anal sex while incarcerated was reported by $23 \%$ of the cases and $9 \%$ of the controls with a history of incarceration (Table 3). Men who reported anal sex while incarcerated were at no greater risk for HIV when controlling for anal sex while not incarcerated (OR, 1.1; 
TABLE 1. Demographic characteristics of cases and controls

\begin{tabular}{lrrrrr}
\hline & \multicolumn{2}{c}{ Cases $(n=305)$} & & \multicolumn{2}{c}{ Controls $(n=305)$} \\
\cline { 2 - 3 } \cline { 6 - 7 } \multicolumn{1}{c}{ Characteristic } & $n$ & $\%$ & & $n$ & $\%$ \\
\hline Marital status & 238 & 78 & & 195 & 64 \\
$\quad$ Single & 8 & 3 & & 16 & 5 \\
Married & 46 & 15 & & 79 & 26 \\
Divorced/separated & 13 & 4 & & 15 & 5
\end{tabular}

OR for single versus married/divorced/separated/other, $2.0 ; 95 \% \mathrm{CI}$, 1.4-2.9

Employment/Status

$\begin{array}{lrrrr}\text { Unemployed } & 272 & 89 & 215 & 71 \\ \text { Employed } & 33 & 11 & 89 & 29 \\ \text { Missing data } & 0 & - & 1 & 0\end{array}$

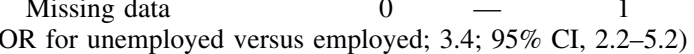

Receiving public assistance or government allotment (welfare) including US Social Security

Yes

No/have not applied

Applied/not received

Missing

$\begin{array}{rrrr}266 & 87 & 206 & 68 \\ 34 & 11 & 93 & 30 \\ 4 & 1 & 5 & 2 \\ 1 & 0 & 1 & 0\end{array}$

OR for on public assistance versus not on public assistance; 3.3;

95\% CI, 2.2-5.0

Annual household income

Less than $\$ 10,000$

$\$ 10,000-24,999$

$\geq \$ 25,000$

Don't know

$\begin{array}{rr}234 & 77 \\ 60 & 20 \\ 9 & \end{array}$

239

50

15

78

16

OR comparing $<\$ 10,000$ versus $\geqslant \$ 10,000,0.9 ; 95 \%$ CI, 0.6-1.4

Education

$\begin{array}{lrrrr}\text { Less than } 12^{\text {th }} \text { grade } & 57 & 19 & 57 & 19 \\ 12^{\text {th }} \text { grade or more } & 245 & 80 & 247 & 81 \\ \text { Missing } & 3 & 1 & 1 & 0\end{array}$

Missing

OR comparing less than 12th with 12 th or more, $1.0 ; 95 \% \mathrm{CI}$, $0.7-1.5$

Sexual orientation

$\begin{array}{lrrrr}\text { Gay } & 134 & 44 & 8 & 3 \\ \text { Bisexual } & 75 & 25 & 24 & 8 \\ \text { Heterosexual } & 90 & 30 & 272 & 89 \\ \text { Other } & 4 & 1 & 1 & 0 \\ \text { Missing } & 2 & 1 & - & -\end{array}$

OR for gay/bisexual versus heterosexual: OR, 20.0; $\overline{9} 5 \%$ CI, $\overline{13} .3$ 29.2

Amounts shown in U.S. dollars.

${ }^{a}$ Defined according to self-reported sexual orientation.

OR, odds ratio; $95 \%$ CI, 95\% confidence intervals.

95\% CI, 0.6-2.2). In an analysis of only those men with a history of incarceration $(n=332), 45 \%$ reported anal sex with men outside the incarcerated setting and $16 \%$ reported anal sex inside the incarcerated setting. The smaller percentage reporting anal sex during incarceration compared with anal sex while in the community was consistent for men of all sexual orientations with a history of incarceration.

Of all men who reported anal sex while incarcerated, $3(9 \%)$ of the cases and $3(18 \%)$ of the controls reported having had anal sex for the first time while incarcerated. Of those reporting anal sex during incarceration $(n=$ 52), $90 \%$ reported having never used a condom during anal sex while incarcerated compared with $42 \%$ who
TABLE 2. Incarceration histories

\begin{tabular}{|c|c|c|c|c|}
\hline & \multicolumn{2}{|c|}{ Cases } & \multicolumn{2}{|c|}{ Controls } \\
\hline & $n$ & $\%$ & $n$ & $\%$ \\
\hline \multicolumn{5}{|c|}{$\begin{array}{l}\text { Incarceration in jail, detention center, or prison between } 1978 \\
\text { and first positive HIV test result }{ }^{a}\end{array}$} \\
\hline Yes & 151 & 50 & 181 & 59 \\
\hline No & 154 & 50 & 124 & 41 \\
\hline \multicolumn{5}{|c|}{$\begin{array}{l}\text { OR comparing those with a history of incarceration to those with } \\
\text { no history of incarceration, } 0.7 ; 95 \% \mathrm{CI}, 0.5-0.9\end{array}$} \\
\hline \multicolumn{5}{|c|}{$\begin{array}{l}\text { Number of incarcerations between } 1978 \text { and first positive HIV } \\
\text { test result }{ }^{a, b}\end{array}$} \\
\hline$\geq 3$ & 66 & 44 & 82 & 45 \\
\hline $1-2$ & 85 & 56 & 97 & 54 \\
\hline Missing & 0 & 0 & 2 & 1 \\
\hline Mean & 3.6 & & 3.0 & \\
\hline \multicolumn{5}{|c|}{ OR comparing $\geq 3$ to $1-2,0.9 ; 95 \% \mathrm{CI}, 0.6-1.4$} \\
\hline \multicolumn{5}{|c|}{$\begin{array}{l}\text { Number of months in jail or prison between } 1978 \text { and first } \\
\text { positive HIV test result }\end{array}$} \\
\hline$\leq 6$ & 77 & 51 & 71 & 39 \\
\hline $7-24$ & 37 & 25 & 51 & 28 \\
\hline $25-36$ & 16 & 11 & 13 & 7 \\
\hline$>36$ & 21 & 14 & 45 & 25 \\
\hline Missing & 0 & - & 1 & 1 \\
\hline \multicolumn{5}{|c|}{$\chi^{2}$ for linear trend, $10.9 ; p$ value $=.001$} \\
\hline \multicolumn{5}{|c|}{ Time in jail and prison versus jail time only } \\
\hline Prison time and/or jail time & 48 & 32 & 68 & 38 \\
\hline Jail time only & 103 & 68 & 111 & 61 \\
\hline Missing & 0 & 0 & 2 & 1 \\
\hline \multicolumn{5}{|c|}{ OR for prison/jail time versus jail time only, $0.8 ; 95 \%$ CI, $0.5-1.2$} \\
\hline
\end{tabular}

${ }^{a}$ Time period under question for controls is 1978 to first positive HIV test result of the control's matched case.

${ }^{\mathrm{b}}$ Totals for these analyses include only those persons who have been incarcerated between 1978 and their first positive HIV test result.

OR, odds ratio; 95\% CI, 95\% confidence intervals.

reported never using a condom during anal sex while not incarcerated.

A history of oral sex while incarcerated was reported by $25 \%$ of the cases and $4 \%$ of the controls, and men who had had oral sex with another man while incarcerated were at no greater risk for HIV when controlling for oral sex with men while not incarcerated (OR, 1.5; 95\% CI, 0.5-4.1). One case and 2 controls who had had oral sex while incarcerated reported that they had oral sex with men for the first time while incarcerated. Most cases $(89 \%)$ and $71 \%$ of controls who had oral sex while incarcerated reported never using a condom during oral sex while incarcerated.

A small percentage of cases (9\%) and controls (4\%) reported that they had traded sex for drugs, money, or other items while incarcerated. Of those with a history of incarceration, $33 \%$ of the cases and $12 \%$ of the controls reported trading sex for drugs or money while not incarcerated. Of all men with a history of incarceration, 3\% reported having sex with a jail and/or prison guard and $3 \%$ reported forced anal sex while incarcerated. Eleven percent of the cases and 17\% of the controls received tattoos while incarcerated; tattooing while incarcerated 
TABLE 3. Sexual behaviors

\begin{tabular}{|c|c|c|c|c|}
\hline & \multicolumn{2}{|c|}{ Cases } & \multicolumn{2}{|c|}{ Controls } \\
\hline & $n$ & $\%$ & $n$ & $\%$ \\
\hline \multicolumn{5}{|c|}{ Anal sex with a man while not incarcerated } \\
\hline \multicolumn{5}{|c|}{ Self-identified gay/bisexual men } \\
\hline Yes & 203 & 97 & 25 & 78 \\
\hline No & 6 & 3 & 7 & 22 \\
\hline \multicolumn{5}{|c|}{ Self-identified heterosexual men } \\
\hline Yes & 28 & 31 & 43 & 16 \\
\hline No & 62 & 69 & 228 & 84 \\
\hline Missing & 0 & - & 1 & 0 \\
\hline \multicolumn{5}{|c|}{ Anal sex with a man while incarcerated ${ }^{a}$} \\
\hline \multicolumn{5}{|c|}{ Self-identified gay/bisexual men } \\
\hline Yes & 29 & 33 & 10 & 48 \\
\hline No & 58 & 67 & 11 & 52 \\
\hline \multicolumn{5}{|c|}{ Self-identified heterosexual men } \\
\hline Yes & 6 & 10 & 7 & 4 \\
\hline No & 54 & 89 & 152 & 96 \\
\hline Missing & 1 & 2 & 0 & - \\
\hline \multicolumn{5}{|c|}{ OR for anal sex with a man while incarcerated, controlling for anal sex with a man while not incarcerated, $1.1 ; 95 \%$ CI: $0.6-2.2$} \\
\hline \multicolumn{5}{|c|}{ Had anal sex with a man for the first time while incarcerated ${ }^{b}$} \\
\hline Yes & 3 & 9 & 3 & 18 \\
\hline No & 32 & 91 & 14 & 82 \\
\hline \multicolumn{5}{|c|}{ Frequency of receptive anal sex with a man while incarcerated ${ }^{\mathrm{b}}$} \\
\hline Never & 10 & 29 & 14 & 82 \\
\hline Sometimes & 10 & 29 & 0 & - \\
\hline Always & 15 & 43 & 3 & 18 \\
\hline \multicolumn{5}{|c|}{ Condom use frequency during anal sex while incarcerated ${ }^{b}$} \\
\hline Never & 32 & 91 & 15 & 88 \\
\hline Sometimes & 3 & 9 & 1 & 6 \\
\hline Always & 0 & - & 1 & 6 \\
\hline \multicolumn{5}{|c|}{ Number of male anal sex partners while incarcerated } \\
\hline 1 & 11 & 31 & 8 & 47 \\
\hline$>1$ & 24 & 69 & 8 & 47 \\
\hline Missing & 0 & - & 1 & 6 \\
\hline \multicolumn{5}{|c|}{ Oral sex with a man while incarcerated ${ }^{a}$} \\
\hline Yes & 37 & 25 & 7 & 4 \\
\hline No & 114 & 75 & 174 & 96 \\
\hline \multicolumn{5}{|c|}{ OR for oral sex with a man while incarcerated, controlling for oral sex with a man while not incarcerated, $1.5 ; 95 \%$ CI, $0.5-4.1$} \\
\hline \multicolumn{5}{|c|}{ Had oral sex with men for the first time while incarcerated ${ }^{\mathrm{b}}$} \\
\hline Yes & 1 & 3 & 2 & 29 \\
\hline No & 36 & 97 & 5 & 71 \\
\hline Frequency of recepti & & & & \\
\hline Always & 17 & 46 & 0 & - \\
\hline Sometimes & 19 & 51 & 4 & 57 \\
\hline Never & 1 & 3 & 3 & 43 \\
\hline Condom use frequen & & & & \\
\hline Never & 33 & 89 & 5 & 71 \\
\hline Sometimes & 4 & 11 & 1 & 14 \\
\hline Always & 0 & - & 1 & 14 \\
\hline Did you exchange sex & d you & tive & esult? & \\
\hline Yes & 14 & 9 & 7 & 4 \\
\hline No & 136 & 90 & 174 & 96 \\
\hline Missing & 1 & 1 & 0 & - \\
\hline Did you have sex with & & & & \\
\hline Yes & 6 & 4 & 4 & 2 \\
\hline No & 144 & 96 & 175 & 98 \\
\hline Refusal/unknown & 1 & 1 & 1 & 1 \\
\hline While incarcerated, did & & & & \\
\hline Yes & 8 & 5 & 1 & 1 \\
\hline No & 142 & 94 & 177 & 98 \\
\hline Refusal/unknown & 1 & 1 & 3 & 2 \\
\hline
\end{tabular}

${ }^{a}$ Totals include men with a history of incarceration.

${ }^{b}$ The totals for these analyses include only those men who reported oral or anal sex while incarcerated.

OR, odds ratio; $95 \% \mathrm{CI}, 95 \%$ confidence intervals. 
was not associated with an increased OR for HIV (OR, 0.6 ; 95\% CI, 0.3-1.2).

IDU during incarceration was reported by $10(7 \%)$ of the cases and $5(3 \%)$ of the controls (Table 4). Men who had injected drugs while incarcerated were no more likely to have HIV infection compared with men reporting no history of IDU while incarcerated while controlling for IDU while not incarcerated (OR, 1.6; 95\% CI, 0.5-4.9). All persons reporting IDU while incarcerated had injected drugs before incarceration.

Demographic data on marital status, income, receiving governmental financial aid (public assistance), and current employment for HIV-infected persons recruited at each of the three study sites was similar to that for the population-based SHAS sample of AIDS cases.

\section{DISCUSSION}

The goal of this research was to describe high-risk behaviors in incarcerated settings and evaluate the extent to which these behaviors contribute to HIV risk for African-American men treated for HIV at three large county public clinics. Anal sex during incarceration was reported by $23 \%$ of the HIV-infected and $9 \%$ of the uninfected men with any history of incarceration and was a particularly high risk due to extremely infrequent condom use. However, anal sex during incarceration was not associated with HIV infection. In fact, for men with a history of incarceration among all categories of sexual orientation, there was more anal sex with men reported during periods of nonincarceration, suggesting that anal sex with men is more common in the community than in

TABLE 4. Injection drug use

\begin{tabular}{|c|c|c|c|c|}
\hline & \multicolumn{2}{|c|}{ Cases } & \multicolumn{2}{|c|}{ Controls } \\
\hline & $n$ & $\%$ & $n$ & $\%$ \\
\hline \multicolumn{5}{|c|}{ Injected drugs while incarcerated } \\
\hline Yes & 10 & 3 & 5 & 2 \\
\hline No & 295 & 97 & 300 & 98 \\
\hline Missing & 0 & - & 0 & - \\
\hline \multirow{2}{*}{\multicolumn{5}{|c|}{$\begin{array}{l}\text { OR for injected drugs while incarcerated controlling for injection } \\
\text { drug use while not incarcerated, } 1.6 ; 95 \% \text { CI, } 0.5-4.9 \\
\text { Injected drugs for the first time while incarcerated }\end{array}$}} \\
\hline & & & & \\
\hline Yes & 0 & - & 0 & - \\
\hline No & 10 & 100 & 3 & 60 \\
\hline Missing & 0 & - & 2 & 40 \\
\hline \multicolumn{5}{|c|}{ Injected drugs while not incarcerated } \\
\hline Yes & 66 & 22 & 49 & 16 \\
\hline No & 238 & 78 & 256 & 84 \\
\hline Missing & 1 & 0 & 0 & - \\
\hline \multicolumn{5}{|c|}{ OR for injected drugs while not incarcerated, $1.4 ; 95 \%$ CI, $0.9-2.1$} \\
\hline
\end{tabular}

${ }^{a}$ Totals for these analyses include persons who reported injecting drugs while incarcerated.

OD, odds ratio; $95 \% \mathrm{CI}, 95 \%$ confidence intervals. the incarcerated setting for this group of AfricanAmerican men.

The potential for underreporting of sexual behaviors with men during incarceration is present, however, and is likely to be greatest for self-identifying heterosexual men who may not consider sex with other men in the incarcerated setting as gay or bisexual behavior (18). Nevertheless, $31 \%$ of cases and $16 \%$ of controls in our study who self-identified as heterosexual admitted to sex with men while not incarcerated, decreasing to $10 \%$ and $4 \%$, respectively reporting sex with men during incarceration. It is unlikely that self-identifying heterosexual men who admit to sex with men while not incarcerated would underreport sex with men while incarcerated, as it is the behavior, not the environment in which the behavior occurs, that is most likely to be underreported. There is still likely to be underreporting of sexual behaviors with men by self-identifying heterosexual men, however this misclassification is unlikely to differ across the incarcerated or other setting. After consideration of the potential underreporting of sex with men by heterosexually selfidentified men in particular, these data continue to suggest that sex with men decreased during incarceration for this group of African-American men of all sexual orientations.

A small percentage of men reported sex with a jail or prison guard and forced anal sex while incarcerated. There is also likely to be underreporting of these behaviors; however, it is not possible to evaluate the extent of misclassification of these variables in the data. Nevertheless, program evaluation within corrections departments should consider how these events can be prevented.

The lack of an association between IDU and HIV infection in or out of incarceration in our data is consistent with a relatively low seroprevalence among IDUs in LAC (19). The best estimate of seroprevalence among IDUs in LAC (3.0\%) comes from a blinded seroprevalence study of persons seeking care in public STD clinics in 1997 (20). Most men who reported IDU during incarceration in our study group reported practicing IDU in California prisons or LAC jails. A seroprevalence survey of new entrants into the LAC jail system in 1995 reported a $2.7 \%$ seroprevalence (7). The seroprevalence among a sample of men entering the California prison system in 1994 was $2.4 \%$ (8). Among the $17 \%$ of the California prison population surveyed for whom risk behavior data was available, there was a $4.2 \%$ seroprevalence among inmates practicing IDU since 1978 (8). In contrast, Dufour's study of inmates in Québec City (Qc, Canada), which found an association between IDU during incarceration and HIV, reported a $10 \%$ seropreva- 
lence among nonincarcerated IDU population (11). These data emphasize the importance of conducting regional studies of risks associated with behaviors during incarceration due to great variability in seroprevalence among high-risk groups in the communities from which a prison population is derived. Possible directions for future research could include the replication of this study design in an area of the United States with a higher HIV seroprevalence among IDUs.

A history of incarceration was not associated with an increased HIV risk, nor were the number of incarcerations between 1978 and a first positive result to an HIV test. These data show a decreasing HIV risk associated with an increasing number of months incarcerated, in contrast to to findings of Dufour who found no association between time incarcerated and HIV (11), and Martin et al. who reported a positive association between the length of a person's prison term and HIV risk (18). Review of the literature suggests that people with prison time in addition to jail time are at greater risk for HIV due to longer sentences and a greater potential for sex with men $(6,18)$ or IDU while incarcerated $(7)$; however, we found no association in our data between prison time and risk behaviors or HIV risk. This is likely due to the low seroprevalence in incarcerated settings in LAC and California, and as shown in these data, the reduction in risk behaviors during incarceration.

A limitation to this study, as in previous research, were the small number of persons reporting sex and drug use during incarceration, resulting in unstable estimates that should be viewed cautiously. Another study limitation is the relatively low response rate for the cases (47\%), which is predominantly due to limited follow-up contact with patients as permitted by the medical sites and because many eligible participants had previously participated in numerous other studies. However, our generalizability assessment demonstrated comparability between the study group and a population-based sample of African-American men diagnosed with AIDS at the three clinics, suggesting that the men in the study group are representative of African-American men diagnosed with AIDS at the three study clinics.

Other limitations to these data include that the study group is not based on population and therefore not generalizable to the entire population of African-American men in LAC, but only to those African-American men who receive their HIV medical care at public medical centers. In addition, because our HIV-infected men were recruited in a medical setting, we were not able to include those men who rarely seek medical care. As a result, we likely excluded the most disenfranchised HIVpositive African-American men whose incarceration his- tories and risk patterns during incarceration may have been different, which may have contributed to selection bias among the cases. It is also possible that because the controls were recruited in public areas of the neighborhoods of the cases, controls may be more likely to be unemployed or on public assistance than the cases. In contrast, however, a larger percentage of the cases than controls were unemployed and on public assistance.

\section{CONCLUSIONS}

Although these data suggest that anal sex with men is more common in the community than within the incarcerated setting for this group of African-American men, incarcerated populations represent a high-risk group for whom access to prevention messages is limited. Periods of incarceration can be viewed as a unique opportunity to convey prevention messages that focus on high-risk behaviors outside of the incarcerated setting.

Acknowledgments: This research was funded in part by the California State Office of AIDS. We would like to acknowledge James Hill and Christopher Moore for their skilled interviewing techniques; Bobby Gatson, Zhijuan Sheng, and Lilia Espinoza of the HIV Epidemiology Program; Carrie Broadus for input into questionnaire development; and Gordon Bunch, Trista Bingham, Nina Harawa, Paul Simon, Richard Sun, Chuck Henry, George Ayala, Mark Miller and Cynthia Davis for their comments on drafts of this manuscript.

\section{REFERENCES}

1. HIV Epidemiology Program, Los Angeles County Department of Health Services. Advanced HIV Dis (AIDS) Surveill Summ 1999;April 15:1-26.

2. California Department of Corrections. Report of ethnicity. Accessed June 1999. Available at: http://www.cdc.state.ca.us/ factsht.htm

3. U.S. Bureau of the Census, Department of Commerce. Population estimates by state, 1998. Washington DC: U.S. Government Printing Office, 1998.

4. Lester B. The social context of HIV transmission in the AfricanAmerican community. Ethnicity Dis 1993;3:387-94.

5. Dolan K, Wodak A, Penny R. AIDS behind bars: preventing HIV spread among incarcerated drug injectors. AIDS 1995;9:825-32.

6. Brewer TF, Derrickson J. AIDS in prison: a review of epidemiology and preventive policy. AIDS 1992;6:623-8.

7. Los Angeles County Sheriff's Department Medical Services. HIV disease management policies, 1996. Los Angeles: Los Angeles County Sheriff's Department Medical Services, 1996.

8. Ruiz JD, Mikanda J. Seroprevalence of HIV, hepatitis B, hepatitis $C$ and risk behaviors among inmates entering the California correctional system. Sacramento, CA: California Department of Health Services, Office of AIDS, 1996.

9. Kennedy DH, Nair G, Elliott L, Ditton J. Drug misuse and sharing of needles in Scottish prisons. BMJ 1995;302:1507.

10. Bird TG, Gore SM, Cameron S, Ross AJ, Goldberg DJ. Anonymous HIV surveillance with risk factor elicitation at Scotland's largest prison, Barlinnie. AIDS 1995;9:801-8.

11. Dufour A, Alary M, Poulin C, et al. Prevalence and risk behaviors 
for HIV infection among inmates of a provincial prison in Quebec City. AIDS 1996;10:1009-15.

12. Taylor A, Goldberg D, Emslie J, et al. Outbreak of HIV infection in a Scottish prison. BMJ 1995;310:289-92.

13. Power KG, Markova I, Rowlands A, McKee KJ, Anslow PJ, Kilfedder C. Sexual behaviors in Scottish prisons. BMJ 1991;302: 1507.

14. Mahon N. New York inmates' HIV risk behaviors: the implications for prevention policy and programs. Am J Public Health 1996;86:1211-15.

15. Wyatt GE. Examining ethnicity versus race in AIDS related sex research. Soc Sci Med 1991;33:37-45.

16. Diaz T, Chu S, Conti L, et al. Risk behaviors of persons with heterosexually acquired HIV infection in the United States: results of a multistate surveillance project. J Acquir Immune Defic Syndr 1994;7:958-63.

17. SAS, version 6.12. Cary, NC: SAS, 1994

18. Martin V, Bayas JM, Laliga A, et al. Seroepidemiology of HIV-1 infection in a Catalonian penitentiary. AIDS 1990;4:1023-1026.

19. Battjes RJ, Pickens RW, Brown LS. HIV infection and AIDS risk behaviors among injecting drug users entering methadone treatment: an update. J Acquir Immune Defic Syndr Hum Retrovirol 1995;10:90-6.

20. County of Los Angeles, Department of Health Services, HIV Epidemiology Program. An epidemiologic profile of HIV and AIDS in Los Angeles County. Los Angeles: County of Los Angeles, Department of Health Services, HIV Epidemiology Program, 1999. 